\title{
Conserved by evolution, but altered in cancer
}

DOI:

10.1038/nrc2261
Some regions of the genome are $100 \%$ conserved between human, mouse and rat, suggesting that they have important functions. A new study shows that changes in the expression of these so-called ultraconserved regions (UCRs) are associated with various cancers.

Several recent studies have shown that, in addition to classical oncogenes and tumour suppressors, microRNAs (miRNAs) have a role in cancer. However, these are not the only type of non-coding RNA - many other parts of the genome appear to be expressed and functionally important, even though they do not encode protein. UCRs are one particular class of these whose functional importance has been demonstrated through deletion experiments in mice.

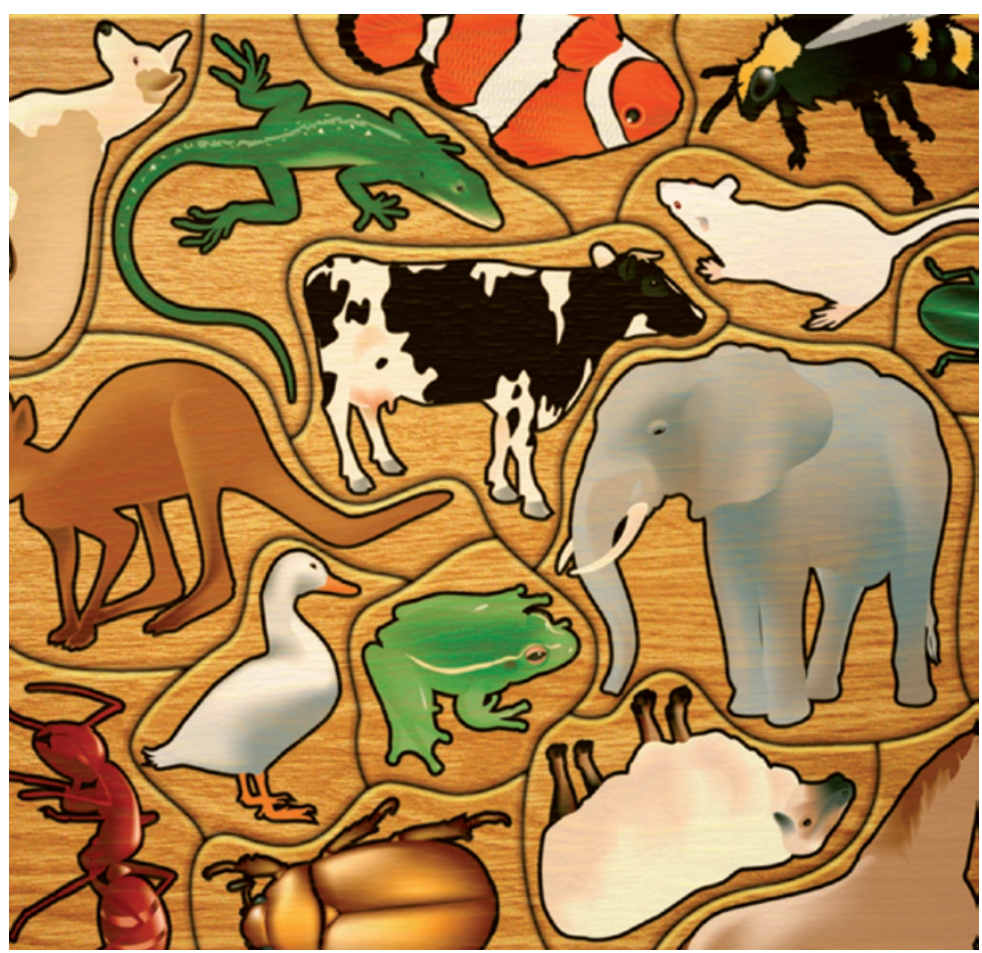

The authors used microarray profiling of UCRs in normal tissue to identify those that were transcribed - T-UCRs. More detailed study using quantitative PCR and northern blots showed that two particular T-UCRs differed significantly in their expression between normal lymphocytes and chronic lymphocytic leukaemia cells. The authors then explored whether T-UCR expression signatures could distinguish between a wide range of normal and cancer cells. Using a panel of 133 cancer samples and 40 corresponding normal controls, they showed that signatures of between 8 and 61 T-UCRs could distinguish specific cancer cells from their normal counterparts.

So what do these T-UCRs do and how are changes in their expression associated with cancer? First, the 